\title{
ARTICLE
}

\section{Cytogenetic analysis of 477 psoriatics revealed an increased frequency of aberrations involving chromosome region $11 \mathrm{q}$}

\author{
Charlotta E nerbäck ${ }^{1,2}$, D eborah H olmqvist ${ }^{1}$, A nnica Inerot ${ }^{2}$, Fredrik E nlund ${ }^{1}$, \\ Lena Samuelsson ${ }^{1}, \AA^{\circ}$ sa Torinsson ${ }^{1}$, Jan Wahlström ${ }^{1}$, G unnar Swanbeck $^{2}$ and \\ Tommy Martinsson ${ }^{1}$
}

${ }^{1}$ D epartments of Clinical G enetics and ${ }^{2} \mathrm{D}$ ermatology, Sahlgrenska U niversity H ospital/Ö stra, G öteborg, Sweden

\begin{abstract}
Psoriasis is an inflammatory skin disorder affecting approximately $3 \%$ of the population. $G$ enetic studies published so far have shown a complex genetic inheritance with heterogeneity and a putative major susceptibility locus in the HLA region on chromosome 6 . We have collected a large amount of material consisting mostly of small nuclear families in order to perform a genome-wide scan for psoriasis-associated genes. In order to focus the scan properly on possible candidate regions, we performed a cytogenetic analysis of 477 unrelated psoriatics. We divided our findings into sporadic, affecting a minor fraction of the cells, and constitutional, ie they were present in all cells examined. We found three cases of balanced translocation, all of which involved chromosome 11q. Two of these had a breakpoint in q12-13, whilst one involved the telomeric part of chromosome $11 \mathrm{q}$. In order to characterise further the breakpoint on 11q12-13, we used bacterial artificial chromosomes (BACs) analysed by fluorescent in situ hybridisation (FISH). We were able to show that the persons had a close, but not identical breakpoints; they were separated by at least $5 \mathrm{cM}$. The major atopy locus is located in this region, as well as a locus for insulin-dependent diabetes mellitus, both being conditions with a pathogenetic mechanism involving antigen presentation.
\end{abstract}

Keywords: psoriasis; cytogenetics; translocation; FISH

\section{Introduction}

Psoriasis is a common skin disorder with a complex genetic background. We have previously published a large population study indicating a recessive mode of inheritance in a Swedish patient sample. ${ }^{1}$ The genetic heterogeneity of this condition is well recognised and, as in several other immunologically mediated diseases,

Correspondence: C E nerbäck, D epartment of Clinical G enetics, Sahlgrenska U niversity Hospital/Ö stra, S-416 85, G öteborg, Sweden. Tel: +46 3134254 64; Fax: +46 318421 60; E -mail: charlotta.enerback@sahlgrenska.se

Received 1 October 1998; revised 20 November 1998; accepted 1 D ecember 1998 there is evidence for a gene within the HLA region. ${ }^{2}$ The genetic linkage analyses presented so far also indicate several possible non-H LA gene locations, all of which have not been confirmed. ${ }^{2-6}$ Two of these studies using a few large pedigrees presented data suggesting a psoriasis susceptibility locus on $17 q$ and $4 q$ respectively. ${ }^{3,4}$ Trembath et al $^{2}$ recently reported a genome scan of 66 affected sib pairs in which four potential psoriasis susceptibility loci were identified, on chromosome $2 p, 6 p$ (HLA region), $8 q$ and $20 p$. Nair et al ${ }^{5}$ confirmed the locus in the HLA region and presented two additional novel loci on chromosome $16 q$ and $20 p$ using $182 \mathrm{sib}$ pairs in a genome-wide scan. 
We have recently confirmed linkage to $6 p$ and $17 q$, but not to $4 q$ in the Swedish patient sample. ${ }^{6}$ H owever, a number of factors make the interpretation of these studies difficult. These include the complex inheritance pattern, differences between population groups, incomplete penetrance, the existence of phenocopies, and the possibility of misdiagnosis. By using a candidate gene approach, ie concentrating attention on genes potentially involved in the pathogenesis of psoriasis due to their function, these problems can be circumvented to some extent.

A nother method of identifying candidate gene regions is to search for chromosomal abnormalities pointing towards a genetic defect involved in the pathogenesis of the disease. Cytogenetic studies have in this way contributed to the identification of several disease genes. Two well-known examples are the Duchenne muscular dystrophe (DMD) gene in $X p 21^{7}$ and the neurofibromatosis type 1 locus in $17 p,{ }^{8}$ where translocations and deletions helped in identifying and cloning the gene. To our knowledge, this approach has so far not been successfully used in genetically complex diseases.

We now report the results from the cytogenetic analysis of 477 unrelated individuals with psoriasis. The main purpose of this study was to focus a genetic linkage analysis of a large psoriasis population on a possible candidate region. We also wanted to estimate the quantity and nature of chromosomal abnormalities present in a psoriasis population. We report on the increased frequency of chromosomal aberrations in the psoriasis patient sample, in particular the involvement of chromosome region $11 \mathrm{q}$ in three constitutionally balanced translocations.

\section{Materials and Methods}

\section{Material}

From a total of 22000 questionnaires sent out to the members of the Swedish Psoriasis A ssociation, data were collected from 14008 families. Blood samples were collected from 104 families where at least two siblings were affected. B oth parents were unaffected in accordance with a recessive model. The material is described in detail elsewhere. ${ }^{1,9}$ To check the diagnosis of the proband, all individuals were examined by one dermatologist (AI) using well established diagnostic criteria. They were also asked about disease history, other diseases and medication. The median age among the probands was 41 years. The 104 families to which they belonged are being used in a current linkage analysis. Cytogenetic analysis was performed in one affected sibling from each family. Blood samples from another unrelated 373 persons with psoriasis were also included in the study.
These patients are used in disequilibrium studies. These persons are also members of the Swedish Psoriasis A ssociation. Cytogenetic analyses were also performed for the parents of three psoriatics with a translocation involving chromosome 11q.

\section{Cytogenetic Analysis}

Cytogenetic analyses were performed in a total of 477 unrelated psoriatics. Spreads from metaphase chromosomes were prepared from cultured peripheral blood lymphocytes according to a modified method described by Johanneson et $\mathrm{al}^{10}$ and analysed using G-banding technique with a resolution approximately at the 400 band level or greater. B etween 5 and 30 mitoses were analysed for each sample (DH).

Isolation of Bacterial Artificial Chromosomes (BACs) The Human DNA pool library, purchased from Research G enetics Inc., H untsville, A L, USA, was screened with PCR assays using primers for the high affinity IgE receptor gene, obtained from the GDB database, and for the polymorphic markers D11S913 and D 11S1314, obtained from the G énéthon map. A ccording to the genetic map, ${ }^{11}$ these markers are located approximately 5 and $10 \mathrm{cM}$ distal to the IgE receptor gene on 11q, respectively. The BAC clones were cultured using standard procedures and extracted using the Q IAGEN Plasmid Purification kit, Hilden, G ermany.

\section{FISH}

The BAC DNA was labelled with Cy3-dCTP and nicktranslated according to the FluoroL ink ${ }^{\mathrm{TM}} \mathrm{Cy}^{\mathrm{TM}} \mathrm{Nick}$ Translation $\mathrm{K}$ it (A mersham LIFE SCIE NCE, Pittsburgh, PA, USA) and was hybridised to metaphase chromosome spreads of cultivated blood lymphocytes according to the procedures of the laboratory. The fluorescence in situ hybridisation was detected with a Zeiss A xiophot microscope and a computerdriven IMAC-CCD colour camera.

\section{Results}

Cytogenetic analyses were performed on one psoriatic sibling in each of 104 families and also on the other 373 unrelated psoriatic persons, in all 185 males and 292 females. The cytogenetic aberrations, divided into constitutional and sporadic, are listed in Table 1 and 2.

Table 1 Constitutional chromosomal aberrations

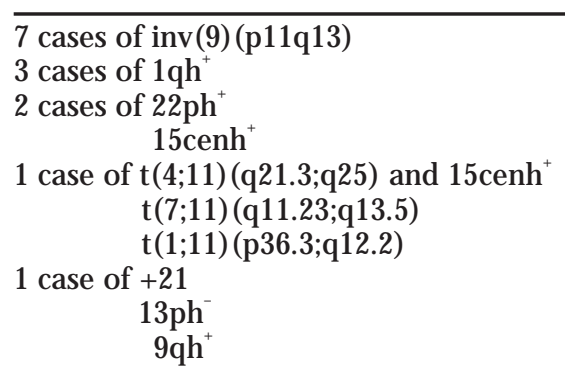


Table 2 Sporadic chromosomal aberrations

\begin{aligned} \hline 1 case of $t(1 ; 12), t(13 ; 15)$ del $1 p, 45, X,-Y \\ t(15 ; 19) \\ t(5 ; 13)(q 13 ; q 34) \\ t(1 ; 15)(q 11 ; q 11) \\ t(X ; 13)(p 11 ; q 11) \\ 1$ case of $t(7 ; 14)(p 11 ; q 11) \\ t(7 ; 14)(p 11 ; q 11) \\ t(7 ; 14)(q 32 ; q 11.2) \\ t(7 ; 14)(q 34 ; q 11) \\ t(7 ; 14)(q 32 ; q 13) \\$ case of del11q13 \\ del $1 q 11 \\$ del $10 q 23 \\$ del $9 q 12 \\$ del $5 p 15 \\$ del $14 q 22 \\$ del $9 p 21 \\$ del $10 q 22 \\ \\ 1$ case of +4 \\ +13 \\ 1 case of inv1p \\ 2 cases of $15 p h^{+} \\ 3$ cases fra $(X)(p 22) \\ 1$ case of fra(2)(q37) \\ fra $(9)(q 31.1) \\ 48 X X, d e l(17)(p 11),+2 m a r \\ 47 X X,+m a r \\ 1$ cases of $45 X / 46 X X / 47 X X X \\ 49 X X X X X \\ 47 X X Y \\ \\ \end{aligned}$

The constitutional abnormalities affect all the 30 analysed cells. In contrast, the sporadic aberrations only affect a minor fraction of the cells, commonly only one (mosaic). Many of the findings can be regarded as normal variations without known pathological significance. In three psoriatics a translocation involving chromosome 11 was found. O ne had the breakpoint in the telomeric part of the chromosome $\mathrm{t}(4 ; 11)(q 21.3 ; q 25)$. The other two had almost identical breakpoints in 11q12-13, ie $t(7 ; 11)(q 11.23 ; q 13.5)$ and $t(1 ; 11)(p 36.3 ; q 12.2)$. These three persons were young, unrelated Swedish psoriatics living in the southern part of Sweden. They all had an early age of onset ( $\mathrm{m}=17$ years) and the diagnosis was confirmed by a dermatologist (AI). They had no history of other immunological diseases and did not take any medication at the time of the cytogenetic analysis. Two of them had one unaffected sibling and the third had no siblings. Because all three of them had unaffected parents and no affected siblings, the segregation of the translocation with the disease could not be followed in these families. For these psoriatics, blood samples were available from the parents. We found that the $t(7 ; 11)$ was a de novo translocation, the $t(1 ; 11)$ was present in the unaffected mother, whereas the $t(4 ; 11)$ was present in the unaffected father.

In order to characterise these translocations further, fluoroscent in situ hybridisation was performed with three bacterial artificial chromosomes (BACs) containing the $\beta$-subunit of the IgE receptor gene (a), genethon marker D11S913 (b) and D11S1314 (c), respectively. The distance between the markers was approximately $5 \mathrm{cM}$. FISH analysis using these BA Cs as probes showed that for the individual with $t(1 ; 11)$, the breakpoint was located between the BACs a and b, and for the individual with $t(7 ; 11)$ it was located distal of probe $c$ (Figure $1 E$ ). A mong the sporadic findings (Table2), we draw attention to an individual with a deletion of 11q13, thus with the same breakpoint as in the two mentioned above, but only in one mitosis. Each of five persons with psoriasis had a translocation $(7 ; 14)$ in one cell; all of them had breakpoints in chromosome 14, (14q11-13). These five individuals did not take any medication, such as methotrexate, nor did they have any other diseases that could explain the finding.

\section{Discussion}

Psoriasis is a skin disorder with an association to the $\mathrm{HLA}$ region. The psoriatic skin lesion is characterised by hyperproliferation of the epidermis together with an inflammatory cell infiltration. O wing to the association with certain HLA antigens, the first attempts to find linkage in population studies were made in this region. By now, several reports confirming the likely presence of a major susceptibility locus in the HLA region have appeared. ${ }^{2,5,6}$ This places psoriasis in the same category as certain other conditions with a strong immunomediated pathogenetic component such as celiac disease, insulin-dependent diabetes mellitus and atopy.

\section{The Breakpoint in 11q25}

Celiac disease is an HLA-associated disease with immunomediated intolerance to gluten resulting in a malabsorption in the small intestine. There is a strong association with the $\mathrm{HLA}$ region but, once again, only part of the familial aggregation is explained by the HLA component. ${ }^{12} \mathrm{~A}$ recently published genome-wide scan indicated the presence of two non-H LA loci, 5pter 
and 11qter. ${ }^{13}$ The marker used for the latter, D 11S934, is mapped to the chromosomal region 11q23-24 according to the GDB (A mplimer) database. Based on these reports, we suggest that the finding of a translocation breakpoint on $11 q 25$ in a psoriatic person could be nonrandom. Trembath et al $^{2}$ also showed suggestive linkage in this region for psoriasis with the maximal $P$ value for the marker D11S910 in the telomeric part of the
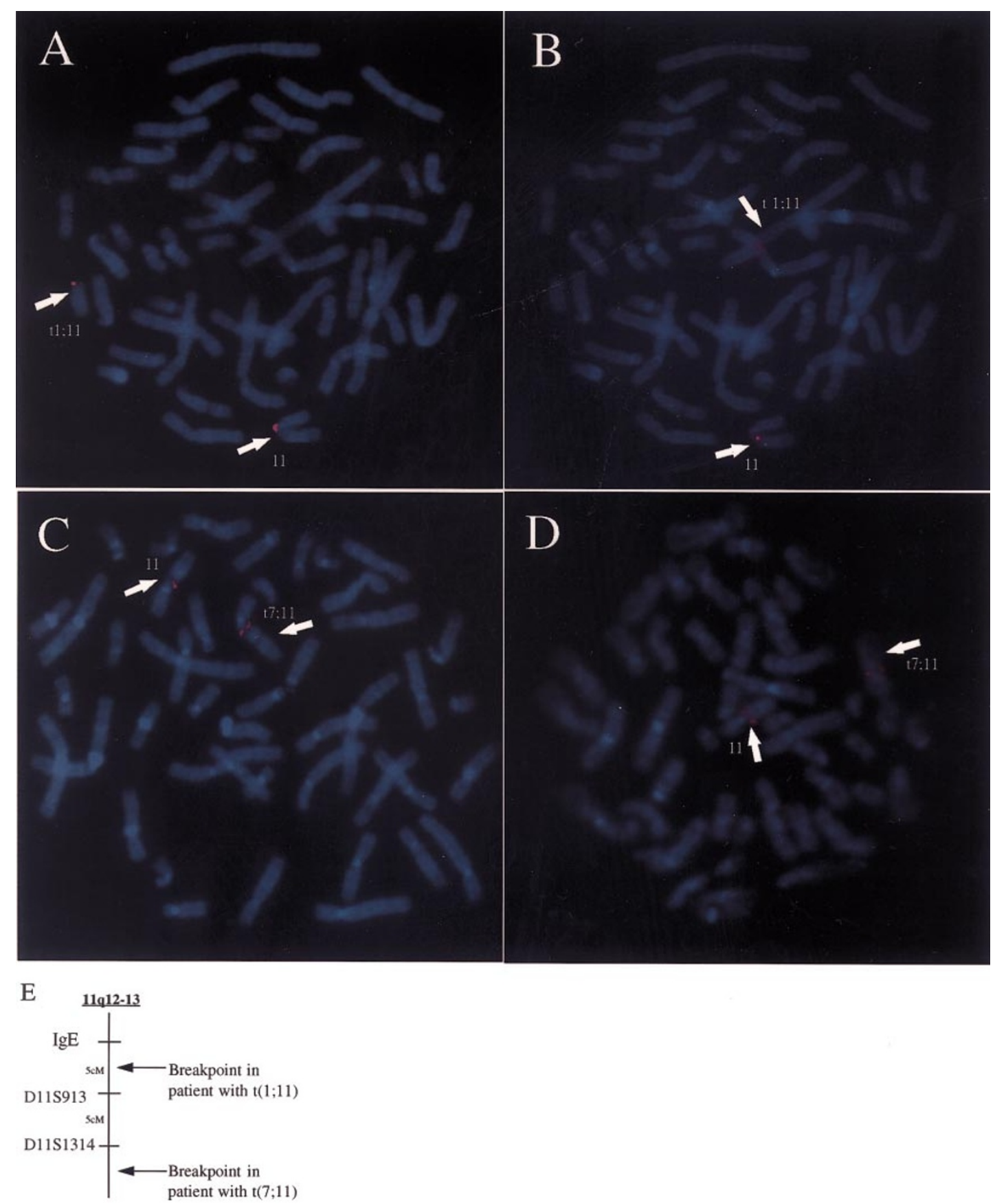

Figure 1 FISH analyses of translocations in psoriatics $\mathbf{A} \quad t(1 ; 11)$ A BAC containing the IgE receptor gene in $11 q$ is hybridised to a metaphase spread and analysed with FISH. The probe is located on $11 q$ and $t(11 p)(1 q)$, thus proximal to the breakpoint. B $t(1 ; 11)$ A BAC containing the marker D 115913 located $5 \mathrm{cM}$ distal to the BAC used in A is used as a probe in the same metaphase. The probe is located on $11 q$ and $t(1 p)(11 q)$, thus distal to the breakpoint. C $t(7 ; 11)$ A BAC containing the IgE receptor gene is hybridised to a metaphase spread and analysed with FISH. The probe is located on $11 q$ and $t(11 p)(7 q)$; thus proximal to the breakpoint. D $t(7 ; 11)$ A BAC containing marker D 11S913 is used as a probe; it is still located proximal to the breakpoint, ie in 11q and $t(11 p)(7 q)$. E L ocation of BACs in relation to chromosomal breakpoints in $11 q$ in two psoriatics. 
chromosome, ie 11q25. There are some indications of a higher prevalence of celiac disease among psoriatics, ${ }^{14}$ which increases the significance of our finding. However, our own data based on linkage analysis of $134 \mathrm{sib}$ pairs do not show any statistically significant $P$ values on chromosome 1lq (manuscript in preparation).

\section{The Breakpoint in 11q12-13}

Insulin-dependent diabetes mellitus is another example of an autoimmune disease with a major susceptibility locus in the $H L A$ region. It is of interest that one of the non-HLA loci, IDDM 4, has been located to chromosome $11 q 13^{15}$

There is also a genetic basis for atopy. A s early as in 1988, Cookson et al reported a locus on chromosome $11 q 12$ using linkage analysis. ${ }^{16}$ There have also been reports of linkage of one of the high affinity IgE receptor genes, located in this region, to clinical atopy, ${ }^{17}$ although several groups later failed to confirm this locus in other populations. ${ }^{18}$ It is regarded as the major atopy candidate gene in this region and it is responsible for the allergic response through the release of inflammatory mediators. We here report two cases of a translocational breakpoint in this subchromosomal region. We used a BAC clone isolated using this gene in a FISH analysis and showed that the gene is located proximal to the breakpoint in both individuals. U sing two BACs containing markers located further downstream, we showed that the breakpoints are closely located, but not identical. A t least $5 \mathrm{cM}$ separates the two breakpoints.

\section{Translocations between Chromosome 7 and 14q11-13}

Five individuals had a translocation $(7 ; 14)$ in one cell out of 30 with the same breakpoint, 14q11-13, where the alpha and delta subunit of the T-cell antigen receptor are located. The significance of the findings, however, is not clear, since there have been reports of sporadic rearrangements involving chromosome 7 and 14 in normal lymphocyte culture. ${ }^{19}$ The T-cells are 'M HC-restricted' in that they identify foreign antigen only in combination with the proper HLA antigen. A dysfunctional T-cell receptor could, in conjunction with a certain HLA antigen ( $\mathrm{Cw} 6$ ), contribute to the inappropriate inflammatory response in psoriasis. We therefore suggest that the significance of our finding cannot be excluded.
Breakpoints in 1p36.3, 4q21.3 and 7q11.23

A two stage genome-scan indicated the presence of a gene locus in 7q11.23 for inflammatory bowel disease. ${ }^{20}$ A ccording to a recent publication on non-H LA loci in autoinflammatory diseases, there are no reports of such loci in $1 p 36.3$ and $4 q 21.3 .^{21}$

There are early reports of chromosomal aberrations in small psoriasis patient samples. A significantly higher frequency of gaps, breaks, dicentric and acentric chromosome fragments was found in 10 psoriatics. ${ }^{22}$ There have also been reports of cytogenetic abnormalities in psoriatic patients treated with methotrexate. ${ }^{23} \mathrm{~A}$ large patient sample is required to overcome the factors that may influence the frequency of these aberrations, such as treatment, infection and the chromosome preparation technique. We examined 5 to 30 cells in each 477 persons with psoriasis and found several cases of constitutional aberrations that are regarded as normal variations. ${ }^{24} \mathrm{~A}$ number of single aberrations occuring only in one cell in one person could be regarded as coincidental or culturing artefacts, because of the very large patient sample examined. A gainst this background of abnormalities, some findings emerge that could point towards a chromosome fragility leading to a translocation. The breakpoint could indicate the location of a disease-causing gene.

There are several hypotheses to explain why balanced translocations have abnormal phenotypes. Submicroscopic deletions in the breakpoints is one explanation and gene disruption resulting in a nonfunctional gene is another. It is also possible that the expression of the translocated gene is altered due to influence of genes in the translocated position. I $f$ the translocation is of causative significance, it implies that a susceptibility gene for psoriasis could be located at the breakpoints on chromosome 11q12-13.

The coincidence of psoriasis and two translocations with an almost identical breakpoint suggests that the translocation is possibly of causal significance for psoriasis in these individuals. There is a possibility that the same gene is affected in both patients. For example, the disruption of a specific gene in one of the patients and a position effect of a translocated segment on the gene in the other patient could lead to the same phenotype due to the inactivation of the same gene. The region 11q12-13 could be considered a region of clustering of non-HLA susceptibility candidate loci in human autoimmune diseases since there are indications 
of an atopy locus, as well as an IDDM locus in this subchromosomal region.

\section{Acknowledgements}

We are grateful for the support of the Swedish Psoriasis A ssociation, the Welander Foundation, the G öteborg M edical Society and the Swedish Medical Research Council (B 96-19X -11246-02B).

\section{References}

1 Swanbeck G, Inerot A, M artinsson T, Wahlström J: A population genetic study of psoriasis. Br J Dermatol 1994; 131: 32-39.

2 Trembath R C, Clough R L, R osbotham J L et al: I dentification of a major susceptibility locus on chromosome $6 p$ and evidence for further disease loci revealed by a two stage genome-wide search in psoriasis. H um M ol G enet 1997; 6: 813-820.

3 Tomfohrde J, Silverman A, Barnes R et al: Gene for familiar psoriasis susceptibility mapped to the distal end of human chromosome 17q. Science 1994; 264: 1141-1145.

4 M atthews D, Fry L, Powles A et al: Evidence that a locus for familial psoriasis maps to chromosome $4 q$. N at $G$ enet 1996; 14: 231-233.

$5 \mathrm{~N}$ air RP, Henseler $\mathrm{T}$, Jenisch $\mathrm{S}$ et al: Evidence for two psoriasis susceptibility loci (HLA and $17 q$ ) and two novel candidate regions ( $16 q$ and $20 p$ ) by genome-wide scan. H um M ol G enet 1997; 8: 1349-1356.

6 Enlund F, Samuelsson L, Enerbäck $C$ et al: A nalysis of three suggested psoriasis susceptibility loci in a large Swedish material; confirmation of linkage to chromosome $6 p$ (HLA -region), and to $17 q$, but not to $4 q$. H um Hered (in press).

7 G reenstein R M, R eardon M P, Chan TS: A n X -autosome translocation in a girl with D uchenne M uscular D ystrophy (DM D ): evidence for D M D gene localization. Pediatr Res 1977; 11: 457.

8 Fountain JW, Wallace MR, Brereton A M et al: Physical mapping of the von Recklinghausen neurofibromatosis region on chromosome 17. A m J G enet 1989; 44: 58-67.

9 Swanbeck G, Inerot A, Martinsson T et al: Genetic councelling in psoriasis. $\mathrm{Br} J$ Dermatol 1997; 137: 939-942.
10 J ohannesson T, H olmqvist D, M artinsson T, Wahlström J : A $n$ improved technique for chromosome preparations from human lymphocytes. H ereditas 1991; 115: 295-297.

11 D ib C, Faure C, Samson N, D rouot A et al: A comprehensive genetic map of the human genome based on 5,264 microsatellites. Nature 1996; 380: 152-154.

12 Petronzelli $F$, Bonamico $M$, Ferrante $P$ et al: Genetic contribution of the $H L A$ region to the familial clustering of coeliac disease. A nn H um G enet 1997; 61: 307-317.

13 G reco L, Corazza G, B abron M C et al: G enome search in celiac disease. A m J G enet 1998; 62: 669-675.

14 Michaelsson G, Gerdén B: How common is gluten intolerance among patients with psoriasis? A cta D erm Ven 1991; 71: 90.

$15 \mathrm{H}$ ashimoto L, H abita C, B eressi J P et al: G enetic mapping of a susceptibility locus for insulin-dependent diabetes mellitus on chromosome 11q. Nature 1994; 371: 8.

16 Cookson W O CM, Sharp P, Faux JA, H opkin J M : L inkage between immunoglobulin $E$ responses underlaying asthma and rhinitis and chromosome 11q. L ancet 1989; I: 1292-1295.

17 Sandford A J, Shirakawa T, M offatt M F et al: L ocalisation of atopy and beta subunit of high-affinity $\mathrm{IgE}$ receptor (FCER 1) on chromosome 11q. Lancet 1993; 341: 332-334.

18 Risch SS, R oitman-Johnson B, G reenberg et al: G enetic analysis of atopy in three large kindreds: no evidence of linkage to D11S97. Clin Exp Allergy 1992; 22: 1070-1076.

19 Wallace $C$, B ernstein R, Pinto M R : Non-random in vitro 7;14 translocations detected in a routine cytogenetic series. H um G enet 1984; 66: 157-161.

20 Satsangi J, Parker M, Louis $M$ et al: Two stage genomewide search in inflammatory bowel disease provides evidence for susceptibility loci on chromosome 3, 7 and 12. Nat G enet 1996; 14: 199-202.

21 Becker K G, Simon R, B ailey-Wilson JE et al: Clustering of non-major histocompatibility complex loci in human autoimmune diseases. Proc Natl A cad Sci USA 1998; 95: 9979-9984.

22 Nielsen J, Zachariae $\mathrm{H}$ : Chromosome aberrations in severe psoriasis. A cta Derm Ven 1973; 53: 192-194.

23 Locher H, Fränz J: Chromosomenveränderungen bei M ethotrexatebehandlungen. M ed Welt 1967; 34: 1956.

24 Jacobs PA, M elville M, R atcliffe S: A cytogenetic survey of 11,680 newborn infants. Ann Hum G enet 1974; 37: 359. 